\title{
Risk Factors for Sustained Cholera Transmission, Juba County, South Sudan, 2014
}

\author{
Thomas T.A. Ujjiga, Joseph F. Wamala, \\ Juma J.H. Mogga, Thabo O. Othwonh, \\ David Mutonga, Asta Kone-Coulibaly, \\ Masood Ali Shaikh, Allan M. Mpairwe, \\ Abubaker Abdinasir, Mohamed A. Abdi, \\ Zabulon Yoti, Olu Olushayo, Pinyi Nyimol, \\ Riek Lul, Richard L. Lako, John Rumunu
}

We conducted a case-control study to identify risk factors for the 2014 cholera outbreak in Juba County, South Sudan. Illness was associated with traveling or eating away from home; treating drinking water and receiving oral cholera vaccination were protective. Oral cholera vaccination should be used to complement cholera prevention efforts.

$\mathrm{C}$ Tholera is an acute diarrheal disease caused by ingestion of food or water contaminated by the bacteria $\mathrm{Vib}$ rio cholerae, of which $\mathrm{O} 1$ is the most common serogroup in Africa $(1,2)$. Although the proportion of global cholera cases reported from sub-Saharan Africa decreased from 93\%-98\% during $2001-2009$ to $44 \%$ in 2013 , cholera remains a major cause of disease epidemics in countries like South Sudan (3). In recent years, 4 major cholera outbreaks have occurred in South Sudan: in 2006, the number of cholera cases totaled 19,277 (case-fatality rate [CFR] 2.9\%); in 2007, cases totaled 22,412 (CFR 1.8\%); in 2008, cases totaled 27,017 (CFR 0.57\%); and in 2009, cases totaled 48,035 (CFR 0.13\%) (4-6; Ministry of Health, South Sudan, unpub. data). A previous case-control study conducted during the 2007 cholera outbreak in Juba County, South Sudan, showed that cholera was associated with being a visitor to Juba, having a water source close to the residence, and treating drinking water (because of inadequate treatment techniques); eating hot food was the only factor significantly associated with a lower risk of cholera (7).

On May 6, 2015, a cholera outbreak was confirmed in Juba County, South Sudan, during a major humanitarian crisis precipitated by political and ethnic tensions that deteriorated dramatically starting in December 2013. Epidemiologic investigations revealed that the outbreak

Author affiliations: Ministry of Health, Juba, South Sudan (T.T.A. Ujjiga, J.J.H. Mogga, T.O. Othwonh, P. Nyimol, R. Lul, R.L. Lako, J. Rumunu); World Health Organization, Juba (J.F. Wamala, D. Mutonga, A. Kone-Coulibaly, M.A. Shaikh, A.M. Mpairwe, A. Abdinasir, M.A. Abdi, Z. Yoti, O. Olushayo)

DOI: http://dx.doi.org/10.3201/eid2110.142051 started on April 23, 2014. We conducted a matched casecontrol study to identify risk factors for, and protective factors against, illness during the 2014 cholera outbreak in Juba County.

\section{The Study}

Cholera case-patients were identified from updated lists from Juba County's 5 cholera treatment centers (CTCs), 2 of which were located in camps for internally displaced persons (IDPs; i.e., persons who have left their homes but stayed within their country's borders). Preventive oral cholera vaccination was conducted in the 2 IDP camps before the outbreak began in Juba. A case-patient was defined as a Juba County resident $\geq 2$ years of age who 1) had an acute illness characterized by $\geq 3$ loose, watery stools within 24 hours or 2) was confirmed to be positive for $V$. cholerae infection by rapid diagnostic testing or culture during the cholera outbreak that began in Juba County on April 23, 2014.

For cholera case-patients enrolled in the study, a control matched by neighborhood, sex, and age was identified and invited to participate in the study. The study team traveled to the case-patient's village and worked with the local village leader or a social mobilization volunteer to identify a matching control from a household within a 100$\mathrm{m}$ radius of the case-patient. A control was a Juba County resident $\geq 2$ years of age with no history of clinical illness or no laboratory evidence of $V$. cholerae infection during this cholera outbreak. To match case-patients and controls by age, age groups of 3-5 years (e.g., 2-4, 5-9, and 65-69 years of age) were used.

A team of 19 trained research assistants administered a pretested, semistructured questionnaire and conducted environmental assessments to evaluate the use of safe drinking water, improved sanitation facilities, personal and food hygiene, and oral cholera vaccination. Using Epi Info (Centers for Disease Control and Prevention, Atlanta, GA, USA), we calculated matched unadjusted and adjusted odds ratios by using bivariate and multivariate models, respectively, to identify risk factors for cholera. The study was approved by the Ministry of Health's ethics committee.

A total of 134 matched pairs of case-patients and controls were enrolled in the study during June 26-July 29 in 2014 (Figure). Of the 134 case-patients enrolled, 9 were confirmed by culture and 104 by a cholera rapid diagnostic test (OnSite Rapid Test; CTK Biotech, San 


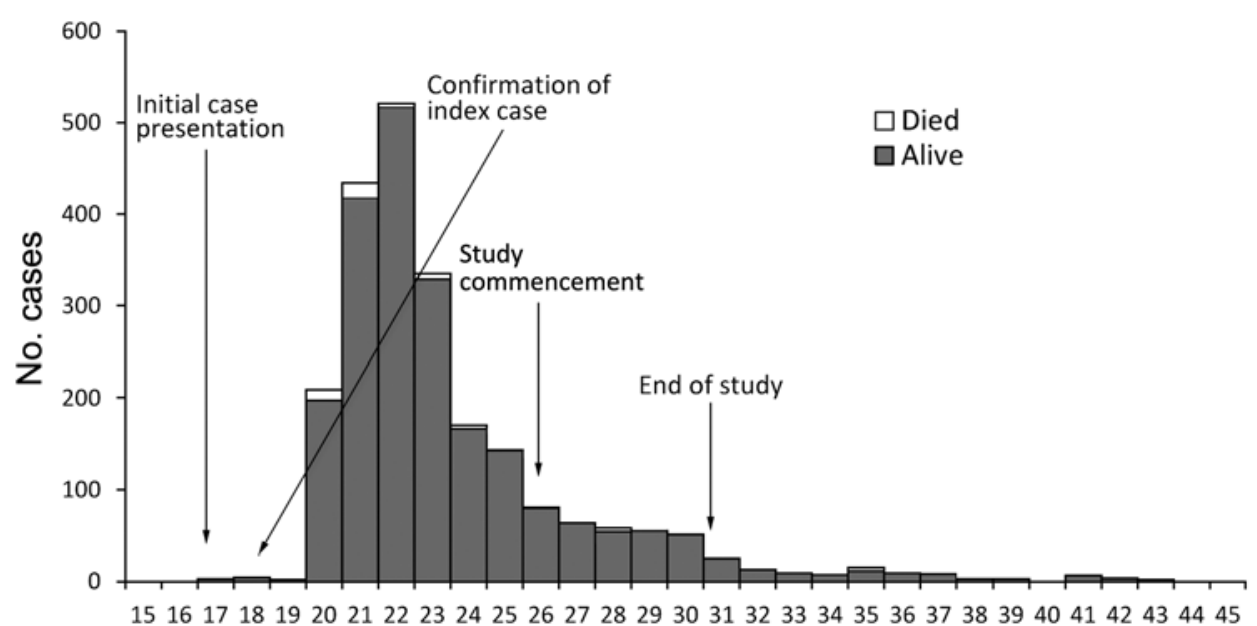

Onset by epidemiologic week, 2014
Figure. Timeline showing number of cholera cases (total cases $=2,260$ ), deaths from cholera (total deaths $=43$; case fatality rate $2.0 \%$ ), and dates of study for cholera outbreak in Juba County, South Sudan, epidemiologic weeks 17-43 (April 23-October 20), 2014.
Diego, CA, USA); the remaining 21 were identified by epidemiologic linkage (i.e., a resident of Juba $\geq 2$ years of age with $\geq 3$ loose stools in 24 hours after the beginning of the cholera outbreak). Eleven cholera casepatients with no matching controls were excluded. Mean delay between admission to the CTC and interview after enrollment in the study was 21 days (range 0-55 days); most case-patients who were interviewed were admitted during the peak transmission phase of the outbreak (Figure). The distribution of age, sex, residence of origin, education level, and occupation were comparable among case-patients and controls (Table 1). Most (118 [88\%]) case-patients enrolled in the study visited the CTC within 1 day of onset of cholera symptoms. All 134 patients had diarrhea; 112 (84\%) had vomiting; 45 (34\%) had abdominal cramps; 37 (28\%) had some dehydration (i.e., any 2 signs of dehydration, including 1 major sign); and $31(23 \%)$ had severe dehydration $(23 \%)$.

Bivariate and multivariate analyses showed that persons who ate food outside their home before illness onset and those who traveled outside their home village (even within the county) before illness onset were significantly more likely to develop cholera (Table 2). Conversely, treating drinking water at home and receiving $\geq 2$ doses of oral cholera vaccine (self-reported) were protective against cholera (Table 2). Eating outside the home as a risk factor in this cholera outbreak is consistent with findings from cholera outbreaks in Uganda and Haiti $(8,9)$. Popular eating places in Juba County included roadside food vendors and restaurants in markets that did not meet minimum food hygiene standards yet remained open during the outbreak because public health inspection of eating establishments and a ban on roadside food vending were not uniformly enforced. Our study identified recent travel to cholera outbreak areas as a risk factor, also a finding consistently associated with cholera spread to new locations during previous cholera outbreaks (10).

Also, as reported in previous cholera outbreaks in South Sudan, Uganda, Haiti, and Zimbabwe, household chlorination of drinking water was associated with significantly lower risk for developing cholera in our study $(7,8,9,11)$. In our study, water samples from case-patient households that did not chlorinate their drinking water showed evidence of contamination with fecal coliforms ( $>10$ counts/100 mL). Similarly, water samples from water storage vessels in 2 case-patient households that did not

\begin{tabular}{|c|c|c|c|}
\hline Characteristic & $\begin{array}{c}\text { Case-patients, } \\
\text { no. }(\%), N=134\end{array}$ & $\begin{array}{l}\text { Controls, no. } \\
(\%), N=134\end{array}$ & $p$ value \\
\hline Age group, y & & & 0.99 \\
\hline 0-9 & $59(45)$ & $60(45.5)$ & \\
\hline $10-19$ & $4(3)$ & $5(3.8)$ & \\
\hline $20-29$ & $28(21.2)$ & $27(20.6)$ & \\
\hline $30-39$ & $20(15.2)$ & $21(16)$ & \\
\hline $40-49$ & $14(10.6)$ & $14(10.7)$ & \\
\hline $50-59$ & $5(3.8)$ & $4(3.1)$ & \\
\hline$\geq 60$ & $1(0.8)$ & $1(0.8)$ & \\
\hline Sex & & & 1.0 \\
\hline$M$ & $67(50)$ & $67(50)$ & \\
\hline $\mathrm{F}$ & $67(50)$ & $67(50)$ & \\
\hline Payam of origin* & & & 0.99 \\
\hline Juba & $47(35.0)$ & $47(35.0)$ & \\
\hline Northern Bari & $36(27.0)$ & $36(27.0)$ & \\
\hline Rejaf & $30(22.4)$ & $30(22.4)$ & \\
\hline Munuki & $6(4.5)$ & $6(4.5)$ & \\
\hline Kator & $7(5.2)$ & $7(5.2)$ & \\
\hline Others & $8(5.9)$ & $8(5.9)$ & \\
\hline Education level & & & 0.85 \\
\hline None & $87(65.4)$ & $88(65.7)$ & \\
\hline Primary/tertiary & $46(34.6)$ & $46(34.3)$ & \\
\hline Employment status & & & 0.18 \\
\hline Unemployed & 100 (81.3) & 112 (86.2) & \\
\hline Employed & $23(18.7)$ & $18(13.8)$ & \\
\hline
\end{tabular}


Table 2. Factors examined by using bivariate and multivariate analyses during cholera outbreak in Juba County, South Sudan, 2014*

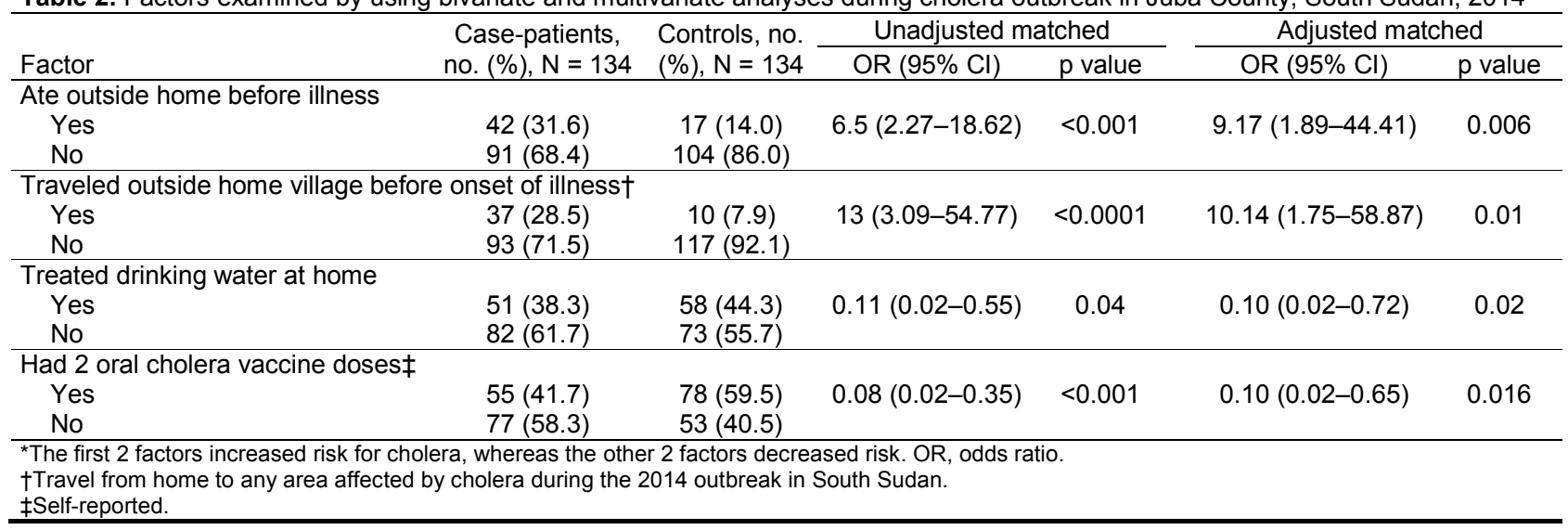

treat their drinking water were contaminated with fecal coliforms ( $>10$ counts $/ 100 \mathrm{~mL}$ ).

Oral cholera vaccination is known to confer protection from cholera (12). We found that oral cholera vaccination was associated with a significantly reduced risk of cholera infection and a vaccine effectiveness of $90 \%$ (Table 2).

Our findings are subject to several limitations that could potentially have confounded our results. These limitations include underreporting of high-risk behaviors, recall bias, potential misclassification of asymptomatic case-patients, narrow age ranges that caused difficulty in identifying matching controls, shared environmental risk factors (e.g., shared water source) for case-patients and controls, unmeasured variables (i.e., factors not measured in this study, such as being an IDP), and loss of oral cholera vaccination cards (i.e., vaccinations were self reported).

\section{Conclusions}

For this cholera outbreak in South Sudan, we found that travel and eating outside the home were risk factors for becoming ill and that treating drinking water at home and getting oral cholera vaccination provided protection against illness. For cholera prevention and control in humanitarian crises, we recommend that global oral cholera vaccine stockpiles be enhanced so that preventive oral cholera vaccination can be used to augment traditional interventions, such as improved access to safe drinking water and public education about risk factors.

\section{Acknowledgments}

All members of the national cholera taskforce are acknowledged for their support of the study. We thank the data collection teams from the Juba Teaching Hospital; the National Public Health Reference Laboratory; the Ministry of Electricity, Dams, Irrigation and Water Resources; and the community health workers at the Juba 3 and Tongping internally displaced persons settlements.
This study was supported by the World Health Organization Country Office for the Republic of South Sudan, with funding from the United States Agency for International Development/ Office of the US Foreign Disaster Assistance; the Central Emergency Response Fund; the Common Humanitarian Fund; and ECHO.

Dr. Ujjiga is a Field Epidemiologist and Acting Director of Epidemic Preparedness and Response at the Ministry of Health in Juba, South Sudan, and is in charge of implementing integrated disease surveillance and response in South Sudan.

\section{References}

1. World Health Organization Global Taskforce on Cholera Control. Cholera outbreak: assessing the outbreak response and improving preparedness. Geneva: World Health Organization; 2004 [cited 2014 Dec 14]. http://whqlibdoc.who.int/hq/2004/WHO_CDS_ CPE ZFk 2004.4 eng.pdf?ua=1

2. World Health Organization. Cholera factsheet no. 107. Geneva: World Health Organization; 2014 Feb [cited 2014 Dec 14]. http://www.who.int/mediacentre/factsheets/fs107/en/

3. World Health Organization. Cholera, 2013. Wkly Epidemiolog Rec. 2014;89:345-56 [cited 2014 Dec 14]. http://www.who.int/ wer/2014/wer8931/en/

4. World Health Organization. Cholera in southern Sudan - update. Geneva: World Health Organization; 2006 March 6 [cited 2014 Dec 14]. http://www.who.int/csr/don/2006_03_06/en/

5. World Health Organization. Cholera in southern Sudan-update 2. Geneva: World Health Organization; 2006 March 22 [cited 2014 Dec 14]. http://www.who.int/csr/don/2006_03_22/en/

6. World Health Organization. Cholera in southern Sudan - update 3. Geneva: World Health Organization; 2006 May 18 [cited 2014 Dec 14]. http://www.who.int/csr/don/2006_05_18a/en/

7. Cholera outbreak - southern Sudan, 2007 . Morb Mortal Wkly Rep. 2009;58:337-41. [cited 2014 Dec 14]. http://www.cdc.gov/mmwr/ preview/mmwrhtml $/ \mathrm{mm} 5813 \mathrm{a} 3 . \mathrm{htm}$

8. Cummings MJ, Wamala JF, Eyura M, Malimbo M, Omeke ME, Mayer D, et al. A cholera outbreak among semi-nomadic pastoralists in north-eastern Uganda: epidemiology and interventions. Epidemiol Infect. 2012;140:1376-85. http://dx.doi.org/10.1017/S0950268811001956

9. Grandesso F, Allan M, Jean-Simon PS, Boncy J, Blake A, Pierre R, et al. Risk factors for cholera transmission in Haiti during inter-peak periods: insights to improve current 
control strategies from two case-control studies. Epidemiol Infect. 2014; 142:1625-35. http://dx.doi.org/10.1017/S0950268813002562

10. Griffith DC, Kelly-Hope LA, Miller MA. Review of reported cholera outbreaks worldwide, 1995-2005. Am J Trop Med Hyg. 2006;75:973-7.

11. Kone-Coulibaly A, Tshimanga M, Shambira G, Gombe NT, Chadambuka A, Chonzi P, et al. Risk factors associated with cholera in Harare City, Zimbabwe, 2008. East Afr J Public Health. 2010;7:311-7.
12. Luquero FJ, Grout L, Ciglenecki I, Sakoba K, Traore B, Heile M, et al. Use of Vibrio cholerae vaccine in an outbreak in Guinea. N Engl J Med. 2014;370:2111-20. http://dx.doi.org/10.1056/ NEJMoa1312680

Address for correspondence: Joseph F. Wamala, World Health Organization Country Office, Juba, South Sudan; email: j_wamala@yahoo.com

\section{facebook}
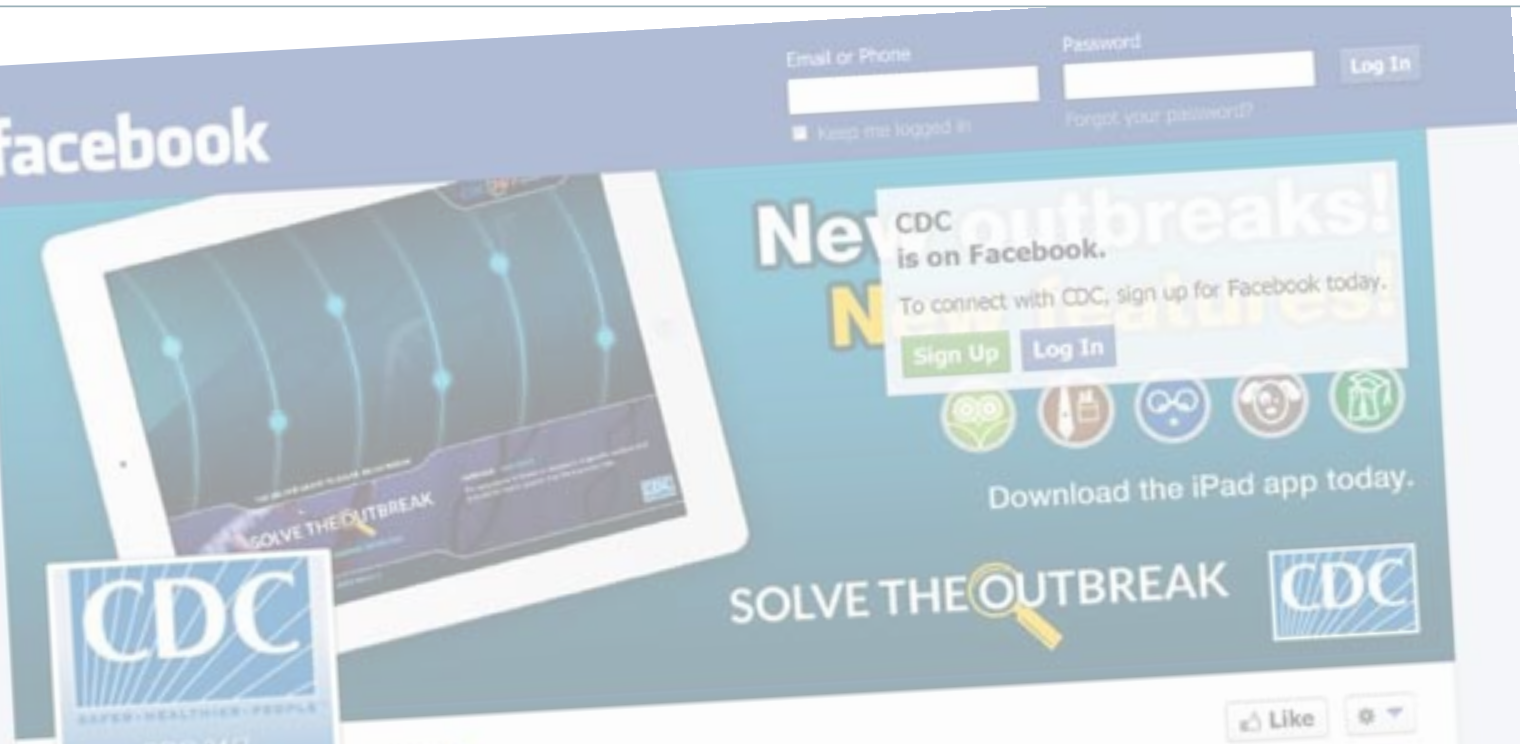

Ne $\operatorname{coc}^{-\infty}$

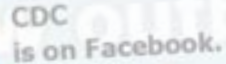

To connect with CoC, sign up for Facebook today. (sign Up $\log$ in $\mathrm{CDC} O$

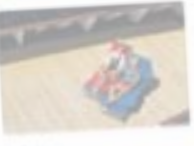

